\title{
EFECTOS DEL ENCALADO Y LA FERTILIZACIÓN NITROGENADA SOBRE EL DESARROLLO DE Oenothera affinis EN UN SUELO AFECTADO POR LA MINERÍA DEL COBRE
}

\section{Effects of liming and nitrogen fertilization on the development of Oenothera affinis in a soil affected by copper mining}

\author{
Victoria Muena ${ }^{1}$, Isabel González ${ }^{1,2}$, Alexander Neaman ${ }^{1,3, *}$ \\ ${ }^{1}$ Facultad de Agronomía, Pontificia Universidad Católica de Valparaíso,
} San Francisco s/n, La Palma, Quillota, Chile. ${ }^{2}$ Doctorado en Gestión Ambiental y Ordenamiento Territorial, Facultad de Geografía, Universidad de Barcelona, Montealegre 6, 08001, Barcelona, España. ${ }^{3}$ Centro Regional de Estudios en Alimentos Saludables (CREAS), Región de Valparaíso, Chile. *Correspondencia: alexander.neaman@ucv.cl

\begin{abstract}
The Puchuncaví valley (central Chile) has been exposed to aerial emissions from the Ventanas copper smelter. Nowadays, soils in the surroundings are sparsely-vegetated, acidic, nitrogen-deficient, and metal-contaminated. Their remediation is needed to reduce environmental risks. Phytostabilization considers the use of plants which are able to store metals in underground tissues, thus reducing their movement from contaminated soils to the air and water. The success of the treatment depends on application of suitable amendments in order to both limit metal solubility in soils and improve soil conditions for the plant development. We evaluated the effects of liming and nitrogen fertilization on cotyledon emergence, plant survival, biomass production and copper accumulation of Oenothera affinis grown in a soil from the Puchuncaví valley, under controlled conditions. Oenothera affinis is a native Chilean perennial herb, it is drought resistant, it is easy to propagate from seeds, and it produces a large biomass. The soil exhibited $\mathrm{pH}$ in salt extract of 4.6 and total copper concentration of $800 \mathrm{mg} \mathrm{kg}^{-1}$. Liming decreased $\mathrm{Cu}$ concentrations and $\mathrm{Cu}^{2+}$ activity in the soil solution, but did not affect cotyledon emergence. None of the plants survived in non-limed soils, while plant survival was $100 \%$ in the soils with near neutral $\mathrm{pH}$. On the other hand, in limed soils, nitrogen fertilization did not affect biomass production and increased copper concentrations in both roots and shoots. It was concluded that liming is sufficient to improve soil conditions for development of $O$. affinis in acidic and metalcontaminated soils, allowing by this mean potential utilization of this species for phytostabilization.
\end{abstract}

Keywords: phytoremediation, phytostabilization, Ventanas smelter, metallophyte, bioavailability, revegetation.

\section{RESUMEN}

El valle de Puchuncaví (Chile central) ha sido expuesto a las emisiones aéreas provenientes de la fundición de cobre Ventanas. Actualmente, los suelos en las cercanías de la fundición presentan una escasa vegetación, son ácidos, deficientes en nitrógeno y contaminados con metales. Para reducir el riesgo ambiental, es necesario remediar estos suelos. La fitoestabilización considera el uso de plantas capaces de 
almacenar los metales en sus estructuras subterráneas, reduciendo así su movimiento desde el suelo contaminado al aire y al agua. El éxito del tratamiento depende de la aplicación de enmiendas adecuadas que reducen la solubilidad de los metales en los suelos y, a la vez, mejoran sus condiciones para el desarrollo de la planta. Se evaluó el efecto del encalado y la fertilización nitrogenada sobre la emergencia de cotiledones, supervivencia, producción de biomasa y acumulación de cobre en Oenothera affinis cultivada en un suelo del valle de Puchuncaví bajo condiciones controladas. Oenothera affinis es una hierba perenne nativa, resistente a la sequía, fácil de propagar a partir de semillas y que produce una gran cantidad de biomasa. Los suelos presentaron un $\mathrm{pH}$ en sal de 4,6 y una concentración de cobre total de $800 \mathrm{mg} \mathrm{kg}^{-1}$. El encalado disminuyó las concentraciones de $\mathrm{Cu}$ y la actividad del $\mathrm{Cu}^{+2}$ en la solución del suelo, pero no afectó la emergencia de cotiledones. Las plantas no sobrevivieron en los suelos no-encalados, mientras que la supervivencia fue del $100 \%$ en los suelos con un $\mathrm{pH}$ cercano al neutro. Por otro lado, en los suelos encalados, la fertilización nitrogenada no afectó la producción de biomasa y aumentó las concentraciones de cobre tanto en las raíces como en los tejidos aéreos. Se concluyó que el encalado es suficiente para mejorar las condiciones del suelo para el desarrollo de O. affinis en suelos ácidos y contaminados por metales, permitiendo así la potencial utilización de esta especie para la fitoestabilización.

Palabras claves: fitorremediación, fitoestabilización, Fundición Ventanas, metalófita, biodisponibilidad, revegetación.

\section{INTRODUCCION}

Los suelos de la zona costera de la comuna de Puchuncaví fueron afectados, por más de treinta años, por las emisiones atmosféricas provenientes de la Fundición Ventanas (Folchi, 2006). Estas emisiones contenían $\mathrm{SO}_{2}$ y material particulado rico en $\mathrm{Cu}$ y otros elementos como As, $\mathrm{Pb}$ y Zn, entre otros (Chiang et al., 1985). Los suelos actualmente son ácidos y presentan altas concentraciones de cobre y otros elementos (Ginocchio, 2000; De Gregori et al., 2003; Neaman et al., 2009). En consecuencia, los ecosistemas asociados a estos suelos se encuentran gravemente deteriorados, lo que se refleja en un paisaje de aspecto semi-desértico de escasa vegetación y evidentes procesos erosivos (Ginocchio, 2000).

En la búsqueda de soluciones para el problema de suelos contaminados por metales, la fitorremediación se perfila como una alternativa económica, de fácil implementación y amigable con el medio ambiente (Salt et al., 1995; Pilon-Smiths, 2004). Esta técnica se define como el uso de plantas para remover, contener o dejar en formas inocuas a los contaminantes del suelo (Pilon-Smiths, 2004). Dentro de las técnicas de fitorremediación, la fitoestabilización está orientada a inmovilizar los metales en el suelo, sin removerlos. Para esto, se utilizan plantas metalófitas excluyentes, es decir, plantas tolerantes capaces de absorber o adsorber los contaminantes en las raíces, sin translocarlos a la parte aérea (Cunningham et al., 1995). De esta forma, la fracción disponible de los metales, es decir, aquella susceptible de ser absorbida por organismos vivos, se mantiene inmovilizada, evitándose la dispersión y el traspaso de metales a la cadena trófica. La técnica permite además mejorar la conservación del suelo y el paisaje al establecer una cobertura vegetal (Wong, 2003; Méndez et al., 2007). 
Las plantas nativas de los sitios a remediar son ideales en cualquier proceso de fitorremediación, ya que han desarrollado los mecanismos apropiados para sobrevivir a las condiciones climáticas y edáficas de dichos ecosistemas. Asimismo, el uso de estas plantas evita la introducción de especies que podrían perjudicar aún más los ecosistemas ya degradados (Yoon et al., 2006; Méndez et al., 2007).

González et al. (2008) realizaron una prospección de especies metalófitas de cobre en el sector de Los Maitenes, en la comuna de Puchuncaví, en la cual se identificaron 22 posibles especies de interés. Entre ellas se destacó la especie nativa Oenothera affinis Cambess (flor de la oración). Esta especie es una hierba anual o perenne (hemicriptófita) (Matthei, 1995), resistente a la sequía, con una alta resistencia a la contaminación metálica y a la acidez del suelo y fácilmente propagable por semillas (González et al., 2008), características que se consideran útiles para los procesos de fitoestabilización.

La disponibilidad de cobre en los suelos de Los Maitenes es alta, lo que se debe a su extrema acidez ( $\mathrm{pH}$ en $\mathrm{KNO}_{3}$ en el rango de 3,9-5,9, González et al., 2008). Además, los suelos presentan una clara deficiencia de nitrógeno (Ginocchio, 2000). Estas condiciones provocan que las plantas que crecen en el sector, a pesar de su tolerancia, presenten bajos porcentajes de germinación, síntomas de toxicidad y un escaso desarrollo de biomasa. Este desarrollo deficiente de las especies que crecen en estos suelos ha sido corroborado mediante ensayos preliminares, comparando su crecimiento con el de plantas que crecen en suelos no afectados por contaminación cúprica.

Para mejorar el desarrollo de las plantas y con ello la eficiencia de la fitoestabilización, es posible adicionar enmiendas al suelo contaminado, previo al establecimiento de las plantas. Los fertilizantes nitrogenados, por ejemplo, incrementaron significativamente la producción de biomasa en plantas de Brassica juncea que se desarrollaron en suelos contaminados por cobre proveniente de una fundición, sin aumentar las concentraciones del metal en los tejidos aéreos (Wu et al., 2004). Por otro lado, la aplicación de cal a suelos ácidos aumenta el $\mathrm{pH}$, provocando la sorción del cobre a las partículas del suelo o su precipitación, con lo que su fracción biodisponible disminuye (Khan y Jones, 2008; Bravin et al., 2009). Este efecto, denominado inmovilización, disminuye la acumulación de metales en las plantas, mejorando de esta forma su germinación y desarrollo (Tyler y Olsen, 2001; Khan y Jones, 2008).

Debido a los resultados positivos que la fertilización nitrogenada y el encalado tienen sobre el desarrollo de las plantas en suelos ácidos contaminados con cobre, es posible suponer que el uso de estas enmiendas mejorarían el desarrollo de $O$. affinis, aumentando así la eficiencia de la fitoestabilización. Por lo tanto, el objetivo de este estudio fue evaluar los efectos del encalado y la fertilización nitrogenada sobre el desarrollo de $O$. affinis en el suelo de Los Maitenes, en las cercanías de la fundición de cobre Ventanas. En este estudio, el "desarrollo" comprendió la emergencia de cotiledones, supervivencia, producción de biomasa y acumulación de cobre. Para este fin, se realizaron ensayos de laboratorio bajo condiciones controladas.

\section{MATERIALES Y MÉTODOS}

\section{Muestreo y preparación del suelo}

El suelo se colectó en el sector de Los Maitenes (UTM 268662 E y 6371085 N), a $3 \mathrm{~km}$ al oriente de La Fundición 
Ventanas en la comuna de Puchuncaví, Región de Valparaíso (Figura 1). Se utilizó como referencia un suelo no afectado por la minería de cobre, proveniente de Maitencillo, a $11 \mathrm{~km}$ al norte de la fundición (UTM 272447 E y $6382175 \mathrm{~N}$ ). Este suelo es comparable al de Los Maitenes por tener características similares de textura y materia orgánica (Cuadro 1). Ambos suelos son paleodunas estabilizadas (Cosio et al., 2007) y fueron clasificados como Entisoles (Soil Survey Staff, 1999). El suelo de Maitencillo se colectó solamente para corroborar que las características químicas del suelo de Los Maitenes eran indicadoras de contaminación.

Se colectaron suelos superficiales, hasta una profundidad de $20 \mathrm{~cm}$. Los suelos fueron esterilizados con vapor a $70^{\circ} \mathrm{C}$ durante 40 min para eliminar semillas viables (Stuckey et al., 2009) y luego fueron secados en una estufa a $60^{\circ} \mathrm{C}$. Posteriormente, el suelo fue tamizado a $2 \mathrm{~mm}$ y homogeneizado en una betonera recubierta de plástico.

\section{Análisis físico-químico del suelo}

El cobre total se determinó luego de la digestión del suelo, usando ácido nítrico al $65 \%$ y ácido perclórico $70 \%$ (adaptación de Verlinden, 1982).

Para la determinación de $\mathrm{pH}$, cobre intercambiable y $\mathrm{Cu}^{+2}$ libre en los suelos, se utilizó una solución de $\mathrm{KNO}_{3} \quad 0,1 \mathrm{M}$ como extractante (razón suelo:solución de $1: 2,5)$.

Para la determinación de cobre total e intercambiable, se utilizó espectrometría de absorción atómica. Se determinó $\mathrm{pCu}^{+2}$ $\left[\mathrm{pCu}^{+2}=-\log \left(\mathrm{Cu}^{+2}\right)\right]$, donde $\left[\mathrm{Cu}^{+2}\right]$ es la actividad del ión libre $\mathrm{Cu}^{+2}$ ), mediante un electrodo selectivo del ión $\mathrm{Cu}^{+2}$. El electrodo fue calibrado usando soluciones tampón de ácido iminodiacético (Rachou et al., 2007).
El contenido de materia orgánica y concentraciones de nitrógeno, fósforo $\mathrm{y}$ potasio disponibles en el suelo se determinaron por los métodos de rutina (Sadzawka et al., 2006). La textura fue determinada por el método simplificado del hidrométro (Sheldrich y Wang, 1993). Para el aseguramiento y control de calidad de los resultados, se analizó una muestra de referencia para cobre (PACS-2, sedimento marino, del National Research Council of Canada). La diferencia entre el valor obtenido y el certificado fue menor al $10 \%$.

\section{Material vegetal}

La población de semillas de $O$. affinis utilizadas para los ensayos fue colectada de varios individuos presentes en el sector de Los Maitenes (UTM 268662 E; 6371085 N, Figura 1). En un ensayo preliminar realizado en placa Petri, se evaluó la emergencia potencial de los cotiledones de las semillas. Durante 15 días, se evaluó diariamente la emergencia de los cotiledones. El día 6, se observó una emergencia de cotiledones de un $52 \pm 9,4 \%$ y al día 12 del ensayo la emergencia alcanzó un máximo del $71 \pm 13,3 \%$.

\section{Emergencia de cotiledones de $\boldsymbol{O}$. affinis en suelo encalado}

Se prepararon sustratos mezclando el suelo de Los Maitenes previamente esterilizado y homogeneizado con distintos porcentajes de $\mathrm{Ca}(\mathrm{OH})_{2}: \mathrm{t}_{0}=0 \%$ (peso/peso), $\mathrm{t}_{1}=0,05 \%, \mathrm{t}_{2}=0,1 \%, \mathrm{t}_{3}=0,2 \%$, $\mathrm{t}_{4}=0,3 \%$ y $\mathrm{t}_{5}=0,5 \%$. Se utilizó $\mathrm{Ca}(\mathrm{OH})_{2}$ como enmienda, al igual que en otros estudios (por ejemplo, Neaman et al., 2009; Stuckey et al., 2009), ya que tiene una reacción alcalinizante más rápida que el $\mathrm{CaCO}_{3}$. Los sustratos se dejaron reaccionar por 30 días en un contenedor sellado en el fondo, humedeciéndolos con 


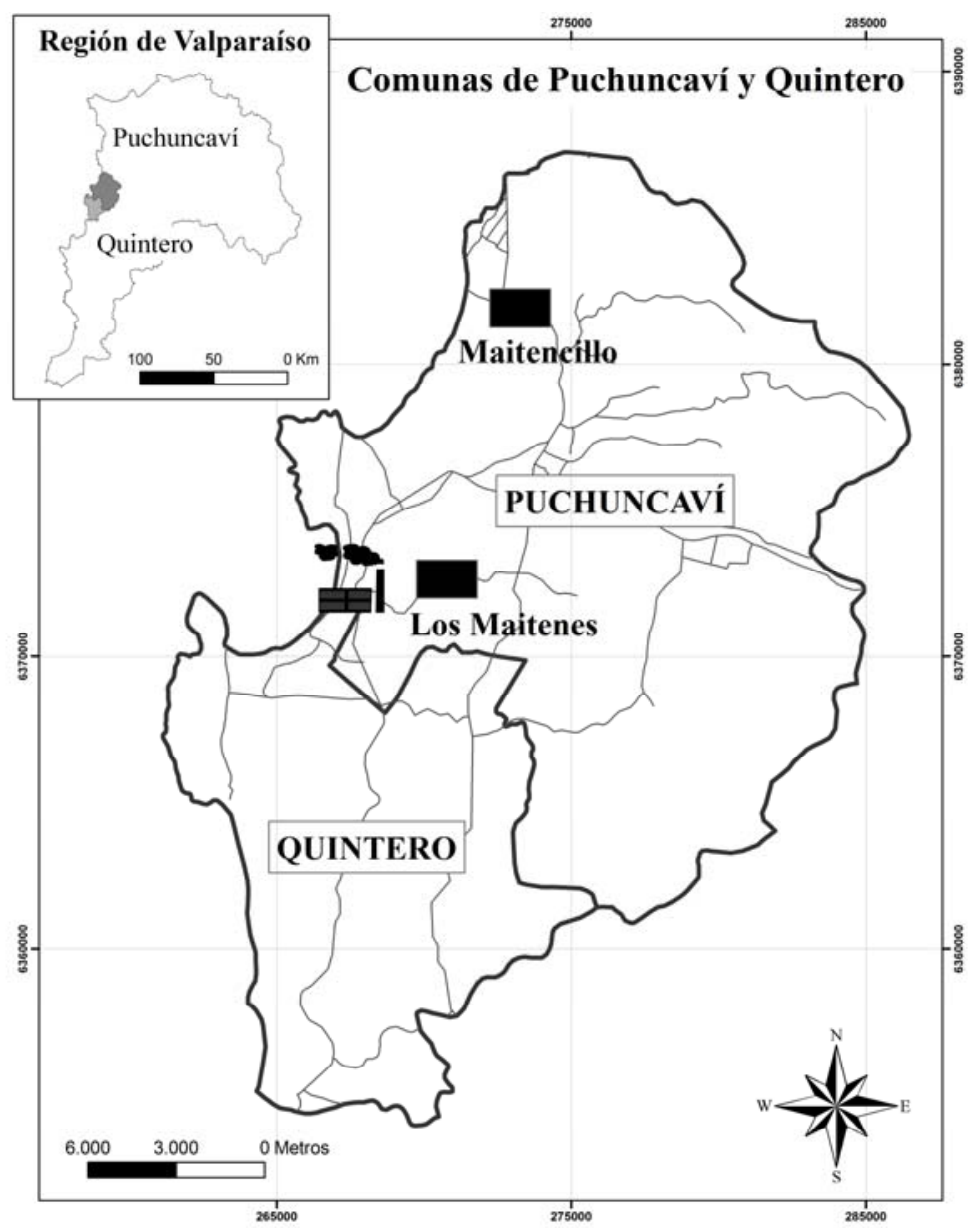

Figura 1. Ubicación de los sectores de muestreo de suelos y semillas.

Figure 1. Location of the soil and seed sampling sites.

agua destilada hasta obtener pasta saturada y mezclándolos cada semana, para luego secarlos a $60^{\circ} \mathrm{C}$. Finalmente, a todos los sustratos se les determinó el $\mathrm{pH}$, $\mathrm{Cu}$ intercambiable y $\mathrm{pCu}^{+2}$.

En los sustratos preparados, se sembraron 10 semillas en contenedores con 120 g de suelo. Se establecieron 6 repeticiones para cada tratamiento.

Los contenedores se mantuvieron en una cámara de crecimiento, con temperatura constante de $23 \pm 1^{\circ} \mathrm{C}$, luminosidad de $1595 \pm 392$ lux, radiación fotosintéticamente activa de 380 a 390 $\mu \mathrm{mol} / \mathrm{m}^{2} / \mathrm{s}$ y $16 / 8$ horas de luz/oscuridad.

Se determinó la capacidad de retención de agua (CRA, ISO 2005). La humedad del suelo se mantuvo aplicando agua destilada cada 3 días, para llegar al 70\% de la CRA.

Las unidades experimentales se distribuyeron al azar dentro de la cámara. El ensayo tuvo una duración de 12 días (en base a resultados de ensayo preliminar), periodo en el cual se evaluó diariamente la emergencia de cotiledones 
en cada tratamiento. La determinación de las diferencias estadísticas entre los tratamientos se realizó mediante análisis de varianza y test de Tukey con una confianza del $95 \%$.

Efecto del encalado y la fertilización nitrogenada en el desarrollo de $O$. affinis

Se prepararon sustratos utilizando el suelo de Los Maitenes, a los cuales se les aplicó nitrato de amonio y/o cal estableciéndose tres tratamientos: $\mathrm{t}_{0}=$ control ( $\mathrm{sin}$ enmiendas), $\mathrm{t}_{1}=\mathrm{Ca}(\mathrm{OH})_{2}, \quad \mathrm{t}_{2}=\mathrm{NH}_{4} \mathrm{NO}_{3}$, $\mathrm{t}_{3}=\mathrm{Ca}(\mathrm{OH})_{2}+\mathrm{NH}_{4} \mathrm{NO}_{3}$. El porcentaje de $\mathrm{Ca}(\mathrm{OH})_{2}$ aplicado al suelo fue de $0,2 \%$ de $\mathrm{Ca}(\mathrm{OH})_{2}$, en base a los resultados del ensayo anterior (se explica en Resultados). La dosis de nitrato de amonio (producto comercial Nitromix, $33 \%$ de N) fue de $43 \mathrm{mg}$ del producto por $\mathrm{kg}$ de suelo, la cual se calculó determinando la diferencia entre una alta concentración de $\mathrm{N}$ en un suelo agrícola (40 mg kg ; Ruiz, 2001) y la concentración de $\mathrm{N}$ disponible en el suelo muestreado (24 $\mathrm{mg} \mathrm{kg}^{-1}$, Cuadro 1).

Los sustratos utilizados se prepararon de la misma forma mencionada en el ensayo anterior. Luego se les determinó $\mathrm{pH}, \quad \mathrm{N}$ disponible, $\mathrm{pCu}^{+2}$ y $\mathrm{Cu}$ intercambiable. Posteriormente, se llenaron contenedores de 1,5 L con 1,3 kg de sustrato, en los cuales se transplantaron 4 plántulas de $O$. affinis previamente germinadas en placas Petri. Cada contenedor se consideró como una unidad experimental y se realizaron 6 repeticiones por tratamiento.

Los contenedores se mantuvieron durante 60 días en una cámara de crecimiento en las condiciones antes mencionadas, modificándose solamente el rango de temperatura $\left(16 / 22^{\circ} \mathrm{C}\right.$, día/noche). La distribución del ensayo en la cámara fue completamente al azar. El riego se realizó con agua destilada cada dos días manteniendo el $70 \%$ de la CRA del suelo. A los 60 días, se determinó la supervivencia de las plantas. Posteriormente, se cosecharon las plantas de cada contenedor, separando tejidos aéreos y raíces. El material vegetal fue lavado con agua potable, luego en una solución de $\mathrm{HCl}$ 0,1 M y agua destilada, para remover las partículas de suelo adheridas al tejido (Tang et al., 1999; Poschenrieder et al., 2001). Las muestras fueron secadas en estufa a $70^{\circ} \mathrm{C}$ por 48 horas y luego se les determinó la biomasa aérea. Finalmente, las muestras fueron trituradas en molino de acero y calcinadas, para posteriormente determinar la concentración de cobre mediante espectrometría de absorción atómica (Sadzawka et al., 2007). Se determinó además la concentración de nitrógeno por método de rutina (Sadzawka et al., 2007).

Para el aseguramiento y control de calidad, se utilizó una muestra de referencia para cobre (tomate 1573a del National Institute of Standards and Technology). La diferencia entre el valor obtenido y el certificado fue menor al $10 \%$. Las diferencias estadísticas entre tratamientos, para las variables analizadas en suelos y material vegetal, se determinaron mediante análisis de varianza y test de Tukey, con una confianza del 95\%.

\section{RESULTADOS}

\section{Propiedades físico-químicas de los suelos estudiados}

La alta concentración total de cobre en el suelo de Los Maitenes, en comparación con el suelo de referencia de Maitencillo (Cuadro 1), indica que existe un impacto de la Fundición Ventanas. La concentración de cobre intercambiable 
Cuadro 1. Características químicas de los suelos estudiados. Se presentan los promedios y desviaciones estándar de las variables analizadas. Concentraciones están expresadas en peso seco.

Table 1. Chemical characteristics of the studied soils. Average and standard deviations of analyzed variables are given. Concentrations are expressed on a dry basis.

\begin{tabular}{lcc}
\hline Variable & Los Maitenes & Maitencillo \\
\hline $\mathrm{Cu}$ total $\left(\mathrm{mg} \mathrm{kg}^{-1}\right)$ & $800 \pm 11$ & $60 \pm 1,7$ \\
$\mathrm{Cu}$ intercambiable $\left(\mathrm{mg} \mathrm{kg}^{-1}\right)$ & $16 \pm 0,10$ & $0,18 \pm 0,02$ \\
$\mathrm{pCu}^{+2}$ & $4,7 \pm 0,003$ & $8,0 \pm 0,56$ \\
$\mathrm{pH}$ & $4,6 \pm 0,01$ & $5,5 \pm 0,22$ \\
$\mathrm{~N}$ disponible $\left(\mathrm{mg} \mathrm{kg}^{-1}\right)$ & $24 \pm 0,56$ & $17 \pm 0,79$ \\
$\mathrm{P}$ disponible $\left(\mathrm{mg} \mathrm{kg}^{-1}\right)$ & $88 \pm 1,6$ & $148 \pm 6,9$ \\
$\mathrm{~K}$ disponible $\left(\mathrm{mg} \mathrm{kg}^{-1}\right)$ & $179 \pm 1,6$ & $130 \pm 0,34$ \\
$\mathrm{~Pb}$ total $\left(\mathrm{mg} \mathrm{kg}^{-1}\right)$ & $103 \pm 6,2$ & $29 \pm 0,43$ \\
$\mathrm{Zn}$ total $\left(\mathrm{mg} \mathrm{kg}^{-1}\right)$ & $220 \pm 9,2$ & $85 \pm 0,72$ \\
Materia orgánica (\%) & $2,3 \pm 0,13$ & $2,1 \pm 0,43$ \\
Arena (\%) & 82 & 89 \\
Limo (\%) & 10 & 6 \\
Arcilla (\%) & 9 & 5 \\
\hline
\end{tabular}

es alta, en comparación con otros suelos contaminados con cobre de la región $\left(0,47 \mathrm{mg} \mathrm{kg} \mathrm{kg}^{-1}\right.$ en el valle de Catemu; Ávila et al., 2007) y en comparación al suelo de Maitencillo. El $\mathrm{pCu}^{+2}$ en el suelo de Los Maitenes es bajo, es decir, la actividad del ion $\mathrm{Cu}^{+2}$ es alta en comparación a otros suelos contaminados por cobre de la región (por ejemplo, 7,9 en el valle de Catemu; Ávila et al., 2009) y en comparación al suelo de Maitencillo. El $\mathrm{Cu}^{+2}$ corresponde a la fracción más tóxica de cobre en el suelo; por lo tanto una alta actividad implica una mayor toxicidad (Sauvé et al., 1997). El pH indica que el suelo es muy ácido (según la clasificación de la Junta de Extremadura, 1992). La concentración de $\mathrm{N}$ disponible en el suelo es baja, aunque las concentraciones de fósforo $\mathrm{y}$ potasio disponibles son normales (según la clasificación de Rodríguez 1992 y Ruiz 2001). Las concentraciones totales de $\mathrm{Zn}$ y $\mathrm{Pb}$ en el suelo están bajo las concentraciones máximas aceptables según normativas internacionales (por ejemplo, $300 \mathrm{mg} \mathrm{kg}^{-1}$ para ambos metales según el Council of the European Communities, 1986). Sin embargo, son altos comparados con las concentraciones en el suelo de Maitencillo (Cuadro 1).

\section{Emergencia de cotiledones de $\boldsymbol{O}$. affinis en suelo encalado}

El pH de los suelos de Los Maitenes aumentó proporcional y significativamente con la dosis del encalado en los distintos tratamientos, lográndose un $\mathrm{pH}$ neutro con la dosis de $0,2 \%$ (Cuadro 2). El aumento del $\mathrm{pH}$ provocó un aumento significativo del $\mathrm{pCu}^{+2}$, lo que indica que la actividad del $\mathrm{Cu}^{+2}$ disminuyó. Esta disminución se debe a la competencia entre protones y iones libres de $\mathrm{Cu}^{+2}$ por los sitios de adsorción en la fase sólida del suelo (McBride, 1994). Al disminuir las concentraciones de protones en la solución, aumenta la adsorción de $\mathrm{Cu}^{+2}$, disminuyendo su actividad.

El aumento del pH también causó la disminución significativa de la concentración de cobre intercambiable en los sustratos, aunque no hubo diferencias significativas entre todos los tratamientos (Cuadro 2). A partir de la dosis de $0,1 \%$ del encalado, el cobre intercambiable fue similar entre los tratamientos. Esto se debe al aumento en la solubilidad de la materia orgánica por sobre $\mathrm{pH} 6$ (Ashworth y Alloway, 2008).

La materia orgánica disuelta se compleja fácilmente con el cobre, formando compuestos estables (Tack y Verloo, 1995). A causa de esto, en general el $\mathrm{Cu}$ intercambiable es bajo en suelos neutros, pero aumenta tanto en suelos ácidos como básicos, 
Cuadro 2. Características químicas de los sustratos utilizados en el ensayo de emergencia de cotiledones. Se presentan los promedios y desviaciones estándar de las variables analizadas. Letras distintas en la misma columna indican diferencias significativas entre los tratamientos $(\mathrm{p} \leq 0,05)$.

Table 2. Chemical characteristics of the substrates used in the cotyledon emergence test. Average and standard deviations of analyzed variables are given. Different letters in the same column indicate significant differences between the treatments. $(\mathrm{p} \leq 0.05)$

\begin{tabular}{cccc}
\hline $\begin{array}{c}\mathbf{C a}(\mathbf{O H})_{2} \\
\text { \% }\end{array}$ & pH & pCu & $\begin{array}{c}\text { Cu intercambiable } \\
\text { mg kg }^{-1}\end{array}$ \\
\hline $\mathrm{t}_{0}=0$ & $4,5 \pm 0,08 \mathrm{a}$ & $4,7 \pm 0,24 \mathrm{a}$ & $16,2 \pm 2,47 \mathrm{a}$ \\
$\mathrm{t}_{1}=0,05$ & $5,3 \pm 0,08 \mathrm{~b}$ & $5,3 \pm 0,05 \mathrm{~b}$ & $4,1 \pm 1,49 \mathrm{~b}$ \\
$\mathrm{t}_{2}=0,1$ & $6,0 \pm 0,04 \mathrm{c}$ & $6,2 \pm 0,05 \mathrm{c}$ & $1,7 \pm 0,24 \mathrm{bc}$ \\
$\mathrm{t}_{3}=0,2$ & $6,9 \pm 0,05 \mathrm{~d}$ & $7,6 \pm 0,07 \mathrm{~d}$ & $1,0 \pm 0,15 \mathrm{c}$ \\
$\mathrm{t}_{4}=0,3$ & $7,5 \pm 0,03 \mathrm{e}$ & $8,7 \pm 0,05 \mathrm{e}$ & $1,4 \pm 0,14 \mathrm{c}$ \\
$\mathrm{t}_{5}=0,5$ & $8,5 \pm 0,09 \mathrm{f}$ & $10,2 \pm 0,08 \mathrm{f}$ & $2,7 \pm 0,50 \mathrm{bc}$ \\
\hline
\end{tabular}

exhibiendo una forma de $U$ en una relación de $\mathrm{pH}$ versus solubilidad de $\mathrm{Cu}$ (McBride 1994, Ashworth y Alloway 2008).

No se observó un efecto significativo del $\mathrm{pH}$ del sustrato sobre el porcentaje final de emergencia de cotiledones en $O$. affinis al día $12(49 \pm 14,7 \%, \mathrm{p}=0,81)$. La dosis de $0,2 \%$ logró un $30 \%$ de aparición de cotiledones en un promedio de 5,6 días, tiempo menor al necesario en otras dosis, por ejemplo 8,3 días en el control (Figura 2). Sin embargo, las diferencias entre tratamientos no fueron significativas $(\mathrm{p}=0,11)$.

Se seleccionó la dosis de $0,2 \%$ para el siguiente ensayo, debido a que con esta dosis se alcanza la neutralidad del suelo. A mayores dosis de encalado es posible que la planta presente deficiencias de hierro y/o zinc debido a la baja disponibilidad de estos elementos en suelos alcalinos (Navarro y Navarro, 2003).

\section{Efecto del encalado y la fertilización nitrogenada sobre el desarrollo de $\mathbf{O}$. affinis}

Como resultado de la aplicación de la dosis de $0,2 \%$ de cal al suelo, el $\mathrm{pH}$ en los suelos de los tratamientos de $\mathrm{Ca}(\mathrm{OH})_{2}$ y $\mathrm{Ca}(\mathrm{OH})_{2}+\mathrm{NH}_{4} \mathrm{NO}_{3}$ aumentó a valores cercanos al neutro (pH 6,7, Cuadro 3). En los tratamientos $\mathrm{NH}_{4} \mathrm{NO}_{3} \quad \mathrm{y}$ $\mathrm{Ca}(\mathrm{OH})_{2}+\mathrm{NH}_{4} \mathrm{NO}_{3}$, la concentración de nitrógeno disponible aumentó respecto al control y alcanzó valores considerados como altos (> $40 \mathrm{mg} \mathrm{kg}^{-1}$; Ruiz, 2001; Cuadro 3).

Luego de 60 días desde el inicio del ensayo, las plantas no sobrevivieron en los tratamientos control y $\mathrm{NH}_{4} \mathrm{NO}_{3}$, mientras que en los tratamientos $\mathrm{Ca}(\mathrm{OH})_{2}+\mathrm{NH}_{4} \mathrm{NO}_{3}$ y $\mathrm{Ca}(\mathrm{OH})_{2}$ se observó un $100 \%$ de supervivencia. En consecuencia, no fue posible diferenciar estadísticamente los efectos de todos los tratamientos sobre la planta, sino solamente las diferencias entre los tratamientos $\mathrm{Ca}(\mathrm{OH})_{2}+\mathrm{NH}_{4} \mathrm{NO}_{3}$ y $\mathrm{Ca}(\mathrm{OH})_{2}$. Entre estos tratamientos, no se encontraron diferencias significativas respecto a la producción de biomasa de $O$. affinis. Sin embargo, se observaron diferencias significativas entre estos dos tratamientos en relación a las concentraciones foliares de cobre y nitrógeno (Cuadro 4).

En el tratamiento $\mathrm{Ca}(\mathrm{OH})_{2}+\mathrm{NH}_{4} \mathrm{NO}_{3}$, se observó un aumento de la concentración de nitrógeno en la planta, en comparación al tratamiento con $\mathrm{Ca}(\mathrm{OH})_{2}$ (Cuadro 4). Esto era esperable por la mayor disponibilidad del elemento en el suelo. Sin embargo, el aumento de N foliar no generó un aumento significativo de biomasa, al contrario de lo reportado por Wu et al. (2004) en 


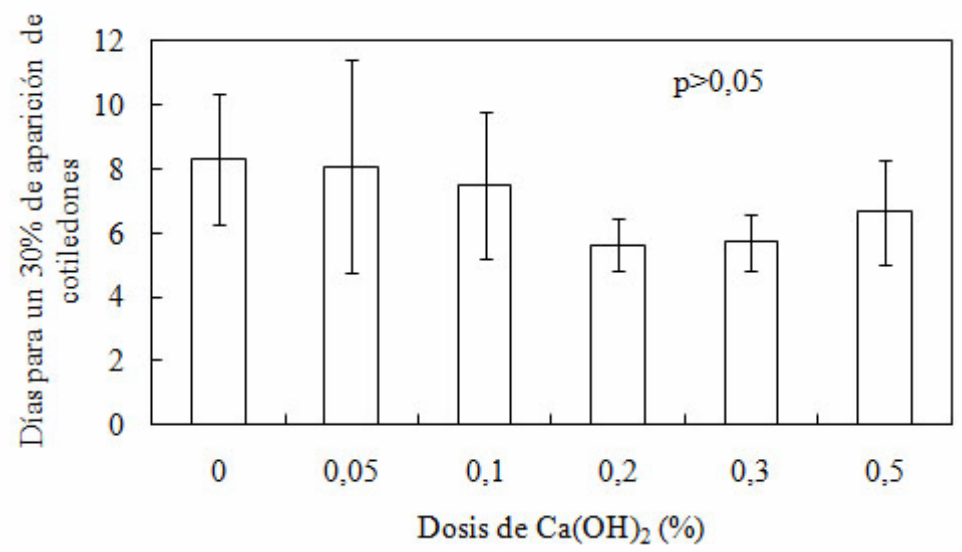

Figura 2. Efecto de la dosis de encalado sobre el tiempo necesario para lograr un $30 \%$ de emergencia de cotiledones de O. affinis. Las barras verticales representan la desviación estándar de las medias.

Figure 2. Effect of lime concentration on the time required to obtain a $30 \%$ of cotyledon emergence of $O$. affinis. Vertical bars represent the standard deviation of the means.

Cuadro 3. Características químicas de los sustratos utilizados para ensayo de desarrollo de $O$. affinis. Se presentan los promedios y desviaciones estándar de las variables analizadas.

Table 3. Chemical characteristics of the substrates used in the O. affinis development test. Average and standard deviations of analyzed variables are given.

\begin{tabular}{|c|c|c|c|c|}
\hline \multirow[t]{2}{*}{ Tratamiento } & \multirow[t]{2}{*}{ pH } & \multirow[t]{2}{*}{$\mathbf{p C u}$} & Cu intercambiable & $\mathrm{N}$ disponible \\
\hline & & & \multicolumn{2}{|c|}{$\mathrm{mg} \mathrm{kg}^{-1}$} \\
\hline Control & $4,6 \pm 0,01$ & $3,4 \pm 0,003$ & $19 \pm 0,10$ & $24 \pm 0,6$ \\
\hline $\mathrm{Ca}(\mathrm{OH})_{2}$ & $6,6 \pm 0,02$ & $6,4 \pm 0,05$ & $1,5 \pm 0,12$ & $24 \pm 1,1$ \\
\hline $\mathrm{NH}_{4} \mathrm{NO}_{3}$ & $4,7 \pm 0,09$ & $3,4 \pm 0,001$ & $21 \pm 0,41$ & $51 \pm 6,2$ \\
\hline $\mathrm{Ca}(\mathrm{OH})_{2}+\mathrm{NH}_{4} \mathrm{NO}_{3}$ & $6,6 \pm 0,01$ & $6,8 \pm 0,01$ & $1,3 \pm 0,51$ & $42 \pm 2,8$ \\
\hline
\end{tabular}

ensayos similares utilizando Brassica juncea. Los posibles motivos de esta diferencia se discuten a continuación.

La concentración de cobre en las raíces fue mayor en el tratamiento $\mathrm{Ca}(\mathrm{OH})_{2}+\mathrm{NH}_{4} \mathrm{NO}_{3}$ en comparación con el tratamiento $\mathrm{Ca}(\mathrm{OH})_{2}$, aunque esto no se pudo comprobar estadísticamente, debido a que la poca muestra obtenida obligó a unir repeticiones. A nivel foliar, se obtuvo una concentración de cobre significativamente mayor en el tratamiento $\mathrm{Ca}(\mathrm{OH})_{2}+\mathrm{NH}_{4} \mathrm{NO}_{3}$, en relación a $\mathrm{Ca}(\mathrm{OH})_{2}$.

El aumento de la acumulación de cobre en raíces y tejidos aéreos puede deberse a un transporte conjunto del $\mathrm{N}$ y el $\mathrm{Cu}$ en la planta, tal como lo observaron Jarvis 
(1984) y Fernández y Henríquez (1991). Estos autores proponen que existen compuestos solubles de nitrógeno que se forman dentro de las raíces en presencia de cobre, los que sirven como agentes que facilitan el transporte activo del metal hacia el interior de la raíz. Estos complejos son aminoácidos proteicos como la histidina y la nicotianamina (Liao et al., 2000; Pilon-Smiths, 2004).

Una vez dentro de las raíces, el cobre es complejado por otros compuestos denominados "chaperones" (carriers), entre los que se encuentran las proteínas denominadas metalotioneínas y fitoquelatinas. Estos "chaperones" transportan el cobre hacia los tejidos aéreos en una molécula que impide el daño celular (Liao et al., 2003; Sharma y Dietz, 2006). En consecuencia, una mayor absorción de cobre a nivel radicular, gracias a la presencia de los agentes facilitadores, tiene como consecuencia una mayor concentración de cobre a nivel foliar, gracias a la acción de los "chaperones" que lo transportan a los tejidos aéreos. Por otro lado, es posible que la mayor
Acumulación de cobre ligada a la mayor acumulación de $\mathrm{N}$ en el tratamiento $\mathrm{Ca}(\mathrm{OH})_{2}+\mathrm{NH}_{4} \mathrm{NO}_{3}$ haya generado un efecto antagónico entre el aporte beneficioso de nitrógeno y el exceso de cobre producido por el transporte conjunto de ambos elementos. Esto explicaría el porqué no se observó un aumento de la biomasa en el tratamiento con nitrógeno.

Se observa en ambos tratamientos que la concentración de cobre en los tejidos aéreos es entre 7 a 9 veces menor que en las raíces, siendo la concentración en la biomasa aérea muy similar a los valores en una planta normal (3-20 $\mathrm{mg} \mathrm{kg}^{-1}$; Gupta y Gupta, 1998), lo que es deseable para la fitoestabilización. En un ensayo en terreno en el sector de Los Maitenes (datos no publicados), en suelos con concentraciones de cobre, textura y $\mathrm{pH}$ similares a los del presente ensayo, se transplantaron plántulas de $O$. affinis al suelo sin ninguna enmienda, para cosecharlos luego de dos meses de cultivo. El análisis de cobre foliar de las plantas arrojó una concentración de $424 \pm 112 \mathrm{mg} \mathrm{kg}^{-1}$.

Cuadro 4. Efecto del encalado y la fertilización nitrogenada sobre la producción de biomasa y concentraciones foliares de $\mathrm{Cu}$ y $\mathrm{N}$ en $O$. affinis. Se presentan los promedios y desviaciones estándar de las variables analizadas Se presentan los promedios y desviaciones estándar de las variables analizadas. Se indica el nivel de significancia para cada una de las variables.

Table 4. Effects of liming and nitrogen fertilization on the biomass production and shoot concentrations of $\mathrm{Cu}$ and $\mathrm{N}$ in $O$. affinis. Average and standard deviations of analyzed variables are given. Significance level is indicated for each variable.

\begin{tabular}{|c|c|c|c|c|}
\hline \multirow[t]{2}{*}{ Tratamiento } & \multirow{2}{*}{$\begin{array}{c}\text { Biomasa } \\
\text { g }\end{array}$} & Cu foliar & Cu raíz & \multirow{2}{*}{$\begin{array}{c}\mathbf{N} \text { foliar } \\
\text { g kg }^{-1}\end{array}$} \\
\hline & & \multicolumn{2}{|c|}{$\mathrm{mg} \mathrm{kg}^{-1}$} & \\
\hline $\mathrm{NH}_{4} \mathrm{NO}_{3}+\mathrm{Ca}(\mathrm{OH})_{2}$ & $1,79 \pm 0,30^{*}$ & $44 \pm 4,6^{* *}$ & $398 \pm 58 * * *$ & $17 \pm 2,7 * *$ \\
\hline $\mathrm{Ca}(\mathrm{OH})_{2}$ & $1,51 \pm 0,20^{*}$ & $38 \pm 2,3 * *$ & $280 \pm 78 * * *$ & $5,0 \pm 0,7 * *$ \\
\hline
\end{tabular}

$* \mathrm{p}>0,05 ; * * \mathrm{p} \leq 0,05 ; * * *$ No fue posible realizar análisis estadísticos debido a que se debieron unir las repeticiones por la poca masa de la muestra, obteniéndose solo dos réplicas para el análisis. 
Si bien los ensayos no son directamente comparables, estos datos indican que el encalado permitió disminuir la acumulación de cobre en la planta en relación al suelo sin enmiendas. Esta observación es consecuente con la disminución de la concentración de cobre intercambiable y la actividad del ión $\mathrm{Cu}^{+2}$ que se observa en los suelos de los dos tratamientos encalados (Cuadro 3). En consecuencia, es posible afirmar que $O$. affinis es útil para la fitoestabilización, siempre y cuando se utilice en combinación con una enmienda calcárea que permita disminuir las fracciones biodisponibles de cobre en el suelo.

\section{CONCLUSIONES}

La aplicación de $\mathrm{Ca}(\mathrm{OH})_{2}$ a los suelos de Los Maitenes disminuyó las concentraciones de $\mathrm{Cu}$ y la actividad del $\mathrm{Cu}^{+2}$ en la solución. Sin embargo, esto no afectó el tiempo ni el porcentaje de emergencia de cotiledones. Las plantas no sobrevivieron en los suelos no encalados, observándose por el contrario un $100 \%$ de supervivencia en los tratamientos encalados.

En los suelos encalados, la fertilización nitrogenada aumentó la concentración de nitrógeno foliar, pero no tuvo efectos sobre la producción de biomasa foliar de la planta. Por otro lado, la fertilización nitrogenada aumentó las concentraciones de cobre en raíces y en tejidos aéreos, lo que no es deseable para la fitoestabilización. En consecuencia, la aplicación de cal es un tratamiento eficiente y suficiente para mejorar las condiciones del suelo para el desarrollo de Oenothera affinis en suelos ácidos y contaminados por metales, permitiendo así la potencial utilización de esta especie para la fitoestabilización.

\section{AGRADECIMIENTOS}

El presente trabajo fue financiado por los proyectos FONDECYT 1050403 y 1085005. Los autores agradecen los aportes y comentarios realizados por Eduardo Salgado y Eduardo Oyanedel (Pontificia Universidad Católica de Valparaíso).

\section{REFERENCIAS}

Ashworth, D.J., Alloway, B.J. 2008. Influence of dissolved organic matter on the solubility of heavy metals in sewage-sludge-amended soils. Communications in Soil Science and Plant Analysis 39, 538-550.

Ávila, G., Gaete, H., Morales, M., Neaman, A. 2007. Reproducción de Eisenia foetida en suelos agrícolas de áreas mineras contaminadas por cobre y arsénico. Pesquisa Agropecuaria Brasileira 42, 435-441

Ávila, G., Gaete, H., Sauvé, S., Neaman, A. 2009. Organic matter reduces copper toxicity for the earthworm Eisenia foetida in soils from mining areas in central Chile. Chilean Journal of Agricultural Research 69, 252-259.

Bravin, M. N., Martí, A.L., Clairotte, M., Hinsinger, P. 2009. Rhizosphere alkalinization: a major driver of copper bioavailability over a broad $\mathrm{pH}$ range in an acidic, coppercontaminated soil. Plant and Soil 318, 257-268.

Chiang, J., Cornejo, P., López, J., Romano, S. Pascual, J., Cea, M. 1985. Determinación de cadmio, cobre, manganeso, plomo, hierro, zinc y arsénico en sedimento atmosférico, en la zona de Quintero, Chile. Boletín de la Sociedad Chilena de Química 30, 139-158.

Cosio, F., Negrón, M., Gastó, J., Villate, G. 2007. Distritos y Sitios de la Provincia Templada Secoestival Nubosa o Valparaíso: secano de la costa. Revista de la Ciencia del Suelo y Nutrición Vegetal 7, 38-61.

Council of the European Communities. 1986. Council of the European Communities directive of 12 of June on the protection of the environment, and in particular of the soil, when sewage sludge is used in agriculture. Official Journal of European Communities 181, 6-12. 
Cunningham, S., Berti, W.R., Huang, J.W. 1995. Phytoremediation of contaminated soil. Trends in Biotechnology 13: 393-397.

De Gregori, I., Fuentes, E., Rojas, M., Pinochet, H., Potin-Gautier, M. 2003. Monitoring of copper, arsenic and antimony levels in agricultural soils impacted and nonimpacted by mining activities, from three regions in Chile. Journal of Environmental Monitoring 5, 287-295.

Fernández, J., Henríquez, F. 1991. Biochemical, physiological, and structural effects of excess copper in plants. The Botanical Review $57,246-273$.

Folchi, M.A. 2006. Historia ambiental de las labores de beneficio en la minería del cobre en Chile, siglos XIX y XX. Tesis doctoral, Departamento de Economía y de Historia Económica, Universidad Autónoma de Barcelona, Barcelona, España, 727 p.

Ginocchio, R. 2000. Effects of a copper smelter on a grassland community in the Puchuncavi Valley, Chile. Chemosphere 41, 15-23.

González, I., Muena, V., Cisternas, M., Neaman, A. 2008. Acumulación de cobre en una comunidad vegetal afectada por contaminación minera en el valle de Puchuncaví, Chile central. Revista Chilena de Historia Natural 81, 279-291.

Gupta, U.C., Gupta, S.C. 1998. Trace element toxicity relationships to crop production and livestock and human health: implications form management. Communications in Soil Science and Plant Analysis 29, 1491-1522.

ISO. 2005. Soil quality-determination of the effects of pollutants on soil flora-Part 1: Method for the measurement of inhibition of root growth ISO 11269-2. International Organization for Standarization, Geneva.

Jarvis, S.C. 1984. The effects of nitrogen supply on the absorption and distribution of copper in red clover (Trifolium pratense L.) grown in flowing solution culture with a low, maintained concentration of copper. Annals of Botany 53, 153-162.

Junta de Extremadura. 1992. Interpretación de Análisis de Suelo, Foliar y Agua de Riego. Ediciones Mundi-Prensa, Madrid, España, 92 p.

Khan, M., Jones, D. 2008. Chemical and organic immobilization treatments for reducing phytoavailability of heavy metals in copper-mine tailings. Journal of Plant Nutrition and Soil Science 171, 908-916.

Liao, J.P., Lin, X.G., Cao, Z. H., Shi, Y.Q., Wong, M.H. 2003. Interaction between arbuscular mycorrhizae and heavy metals under sand culture experiment. Chemosphere 50, 847853.

Liao, M.T., Hedley, M.J., Woolley, D.J., Brooks, R.R., Nichols, M.A. 2000. Copper uptake and traslocation in chicory (Chichorium intybus L. cv. Grassland Puna) and tomato (Lycopersicon esculentum Mill. cv Rondy) plants grown in NET system II. The role of nicotinamin and histidine in xylem sap copper transport. Plant and Soil 223, 245-254.

Matthei, J. 1995. Manual de las Malezas que Crecen en Chile. Alfabeto Impresores, Santiago. $537 \mathrm{p}$.

McBride, M.B. 1994. Environmental Chemistry of Soils. Oxford University Press, New York, NY., $406 \mathrm{p}$.

Méndez, M., Glenn, E., Maier, R. 2007. Phytostabilization potential of quailbush for mine tailings: Growth, metal accumulation and microbial community changes. Journal of Environmental Quality 36, 245-253.

Navarro, S., Navarro, G. 2003. Química Agrícola: El suelo y los Elementos Químicos Esenciales Para la Vida Vegetal. Ediciones Mundi-Prensa, Bilbao, España, 487 p

Neaman, A., Reyes, L., Trolard, F., Bourrié, G., Sauvé, S. 2009. Copper mobility in contaminated soils of the Puchuncaví valley, central Chile. Geoderma 150, 359-366.

Pilon-Smiths, E. 2004. Phytoremediation. Annual Review of Plant Biology 56, 15-39.

Poschenrieder, C., Bech, J., Llugany, M., Pace, A., Fenés, E., Barceló, J. 2001. Copper in plant species in a copper gradient in Catalonia (North East Spain) and their potential for phytoremediation. Plant and Soil 230, 247-256.

Rachou, J., Gagnon, C., Sauvé, S. 2007. Use of an ion selective electrode for free copper measurements in low salinity and low ionic strength matrices. Environmental Chemistry 4, 90-97.

Rodríguez, J. 1992. Manual de Fertilización. 362 p. Facultad de Agronomía. Pontificia Universidad Católica de Valparaíso, Santiago, Chile. 
Ruiz, R. 2001. Nutrición vegetal, fertilizantes y fertilización. En: Agenda del Salitre. Sociedad Química y Minera de Chile S.A., Santiago, Chile, pp. 175-196.

Sadzawka, A., Carrasco, M., Grez, R., Mora, M., Flores, H., Neaman, A. 2006. Métodos de Análisis Recomendados para los Suelos de Chile. Instituto de Investigaciones Agropecuarias, Santiago, Chile, $164 \mathrm{p}$.

Sadzawka, A., Carrasco, M. A., Demanet, R., Flores, H., Grez, R., Mora, M. L., Neaman, A. 2007. Métodos de Análisis de Tejidos Vegetales. Instituto de Investigaciones Agropecuarias, Santiago, Chile, $140 \mathrm{p}$

Salt, D. E., Blaylock, M., Kumar, P. B., Dushenkov, V., Ensley, B. D., Chet, I., Raskin, I. 1995. Phytoremediation: A novel strategy for the removal of toxic metals from the environment using plants. Biotechnology 13, 468-474.

Sauvé, S., Mc Bride, M.B., Norvell, W.A., Hendershot, W. 1997. Copper solubility and speciation of in situ contaminated soils: effects of copper level, $\mathrm{pH}$ and organic matter. Water, Air and Soil Pollution 100, 133-149.

Sharma, S., Dietz, K.J. 2006. The significance of amino acids and amino acid-derived molecules in plant responses and adaptation to heavy metal stress. Journal of Experimental Botany 57, 711726.

Sheldrich, B.H., Wang, C. 1993. Particle size distribution. In: M. Carter (ed.). Soil Sampling and Methods of Analyisis. Canadian Society of Soil Science. Lewis Publishers, Boca Ratón, Florida, pp: 499-511.

Stuckey, J., Neaman, A., Ravella, R., Komarnei, S., Martínez, C. 2009. Highly charged swelling mica reduces $\mathrm{Cu}$ bioavailability in $\mathrm{Cu}$-contaminated soils. Environmental Pollution 157, 12-16.
Tack, F.M., Verloo, M.G. 1995. Chemical speciation and fractionation in soil and sediment heavy metal analysis: a review. International Journal of Environmental Analytical Chemistry $59,225-238$

Tang, S., Wilke, B.M., Huang, J.W. 1999. The uptake of copper by plants dominantly growing on copper mining spoils along the Yangtze River, the People's Republic of China. Plant and Soil 209, 225-232.

Tyler, G., Olsen, T. 2001. Plant uptake of major and minor elements as influenced by soil acidity and liming. Plant and Soil 230: 307-321.

Soil Survey Staff. 1999. Soil Taxonomy: A Basic System of Soil Classification for Making and Interpreting Soil Surveys. United States Department of Agriculture, Natural Resources Conservation Service, Agriculture Handbook 436. Washington, DC, USA, $2^{\mathrm{a}}$ ed., $871 \mathrm{pp}$.

Verlinden, M. 1982. On the acid decomposition of human blood and plasma for the determination of selenium. Talanta 29, 875-882.

Wong, M.H. 2003. Ecological restoration of mine degraded soils, with emphasis on metal contaminated soils. Chemosphere 50, 775-80.

Wu, L.H., Li, H., Luo, Y.M., Christie, P. 2004.

Nutrients can enhance phytoremediation of copper-polluted soil by Indian mustard. Environmental Geochemistry and Health 26, 331335 .

Yoon, J., Cao, X., Zhou, Q., Ma, L.Q. 2006. Accumulation of $\mathrm{Pb}, \mathrm{Cu}$, and $\mathrm{Zn}$ in native plants growing on a contaminated Florida site. Science of the Total Environment 368, 456-464. 